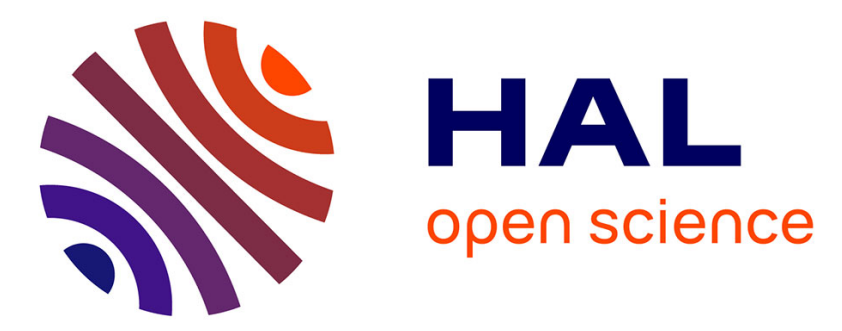

\title{
The density of states of a particular class of topologically disordered structures
}

M. Astier, O. Betbeder-Matibet, D. Calecki, M. Hulin, N. Pottier

\section{To cite this version:}

M. Astier, O. Betbeder-Matibet, D. Calecki, M. Hulin, N. Pottier. The density of states of a particular class of topologically disordered structures. Journal de Physique, 1975, 36 (12), pp.1371-1381. 10.1051/jphys:0197500360120137100 . jpa-00208385

\section{HAL Id: jpa-00208385 https://hal.science/jpa-00208385}

Submitted on 1 Jan 1975

HAL is a multi-disciplinary open access archive for the deposit and dissemination of scientific research documents, whether they are published or not. The documents may come from teaching and research institutions in France or abroad, or from public or private research centers.
L'archive ouverte pluridisciplinaire HAL, est destinée au dépôt et à la diffusion de documents scientifiques de niveau recherche, publiés ou non, émanant des établissements d'enseignement et de recherche français ou étrangers, des laboratoires publics ou privés. 


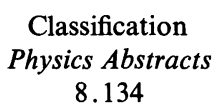

\title{
THE DENSITY OF STATES OF A PARTICULAR CLASS OF TOPOLOGICALLY DISORDERED STRUCTURES
}

\author{
M. ASTIER, O. BETBEDER-MATIBET, D. CALECKI, M. HULIN and N. POTTIER \\ Groupe de Physique des Solides de l'Ecole Normale Supérieure $\left({ }^{*}\right)$, \\ Université Paris VII, 2, place Jussieu, 75005 Paris, France
}

(Reçu le 22 juillet 1975, accepté le 22 août 1975)

\begin{abstract}
Résumé. - On définit une classe de réseaux bidimensionnels de coordinance 3 et une classe de réseaux tridimensionnels de coordinance 4 ; ces réseaux présentent un désordre topologique partiel. Leurs caractéristiques permettent un calcul exact des moyennes d'ensemble des premiers moments. On en déduit la densité d'états moyenne par une méthode de fraction continue; ses traits les plus marquants sont interprétés par référence à des structures ordonnées simples.

Abstract. - A class of 3-fold coordinated 2-dimensional networks, and a class of 4-fold coordinated 3-dimensional networks have been defined, which show partial topological disorder. Their characteristics make it possible to calculate exact average values of the first moments of the density of states over the whole class of structures. The average density of states is then calculated by use of a continued fraction method, and its main features are interpreted by reference to the densities of states of a few related ordered structures.
\end{abstract}

1. Introduction. - The study of topological disorder is of particular importance in connection with the analysis of the electronic properties of perfect covalent semiconductors of constant coordination number $Z$. Within the framework of a simplified LCAO theory, where the constant interaction is restricted to pairs of first neighbour atoms, it has been shown [1-9] that the electronic energy density of states (DOS) can be derived, through a simple analytic transformation, from the eigenvalue distribution of a 1-band hamiltonian with one (say one s) orbital per atom and a constant matrix element (taken as unity through a convenient choice of the energy scale) between every atom and its $Z$ first neighbours.

Such a study, because of computational limitations, has essentially been limited to finite clusters of atoms of given structure [10-14], extended by imposing periodic boundary conditions, tree-like decorations, etc... The DOS $N(E)$ is then generally approached by the local density of states for either one or a few atoms near the center of the cluster.

In this paper, we wish to report some preliminary results which have been obtained adopting a slightly different point of view : by imposing supplementary restrictions on the bonds between atoms, we define a class of networks for which an ensemble average of the DOS can be calculated.

(*) Laboratoire associé au C.N.R.S.
We consider here networks which are :

i) 3-fold coordinated $(Z=3)$ and 2-dimensional, or 4-fold coordinated and 3-dimensional,

ii) alternant (i.e., all closed rings involve an even number of bonds),

iii) only partially disordered so that they keep partial spatial periodicity.

The DOS is approached by the moment technique. The exact ensemble averages of the first moments are calculated (up to 14 non-zero moments for 2-dimensional lattices, and to 9 or 10 non-zero moments for 3-dimensional lattices). The results are discussed by comparison with the DOS of some related ordered structures.

Section 2 is devoted to 2-dimensional 3-fold coordinated networks, which enables us to introduce the main ideas of this work in a simple, easily visualized situation. Section 3 deals with 3-dimensional 4-fold coordinated networks.

2. Two-dimensional systems. -2.1 DesCription OF THE NETWORKS. - An ordered 2-dimensional lattice with $Z=3$ is realized in the honeycomb lattice (HL), the topological properties of which are maintained when it is redrawn as shown in figure $1 a$. It is obvious that it results from the suppression of one out of 4 bonds for every atom in a square lattice, this bond being systematically parallel to the $x$-axis, 




FIG. 1a. - Honeycomb lattice (HL). 1b. - Density of states for the honeycomb lattice.

and, along any chain parallel to the $y$-axis, alternately on one side or the other.

The disordered structures which we consider simply result from a random distribution of the suppressed $x$-bonds between chains, a constant coordination number $Z=3$ being kept for every atom. (Such a random bond suppressing procedure, starting from a periodic lattice, has already been used by L. Gutt$\operatorname{man}[10]$.)

Let us label the atoms by integer coordinates $x=m$ and $y=n$. Along the row $y=n$, the $x$-bonds correspond either to the arrangements $(a)$ or $(b)$ in figure 2 , both of which are periodic along the $x$-direc- tion. To this $n$th row of atoms we associate a network parameter $\varepsilon_{n}$ equal to \pm 1 depending on whether this row is of $(a)$ or $(b)$ type. We can thus define different networks, each of them corresponding to a given distribution of $\varepsilon_{n}^{\prime} \mathrm{s}:$ the honeycomb lattice for instance (Fig. 1a) obviously corresponds to $\varepsilon_{n}=(-1)^{n}$.

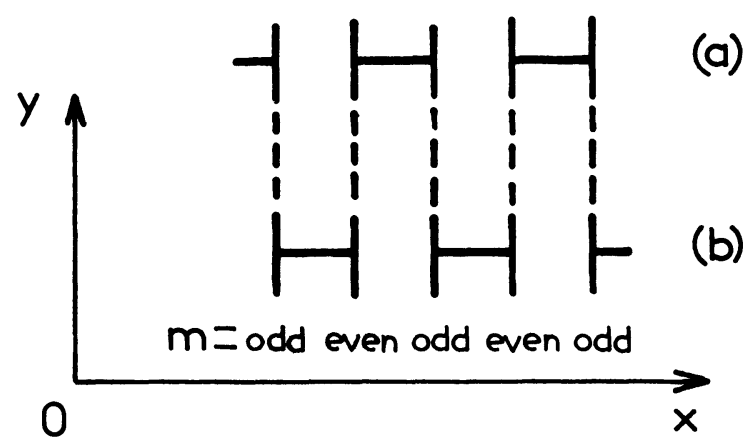

FIG. 2. - The two possible arrangements of bonds along a row of atoms parallel to the $x$-axis.

For disordered networks, we only consider the case where this distribution is completely random, each $\varepsilon_{n}$ taking its two possible values with equal probabilities, with no correlation between $\varepsilon_{n}^{\prime} \mathrm{s}$ for different indices. We call this model the random honeycomb lattice (RHL). All these networks clearly exhibit the same periodicity along the $x$-axis as rows $(a)$ or $(b)$, and their randomness has a 1-dimensional character : we shall thus be able to refer to the results obtained for 1-dimensional systems $[15,16]$ as a guideline through the following.

This periodicity with two adjacent $y$-chains of, say, $N$ atoms as a unit cell results in the possibility of realizing a Bloch transformation of the initial 1-band hamiltonian $\mathcal{H}$ which transforms it in a series of $(2 N \times 2 N)$ subhamiltonians $\operatorname{He}(\varphi)$ where $\varphi$ is analogous to a wave-vector along the $x$-axis. $\mathcal{H e}(\varphi)$ can be readily seen to be of the form :

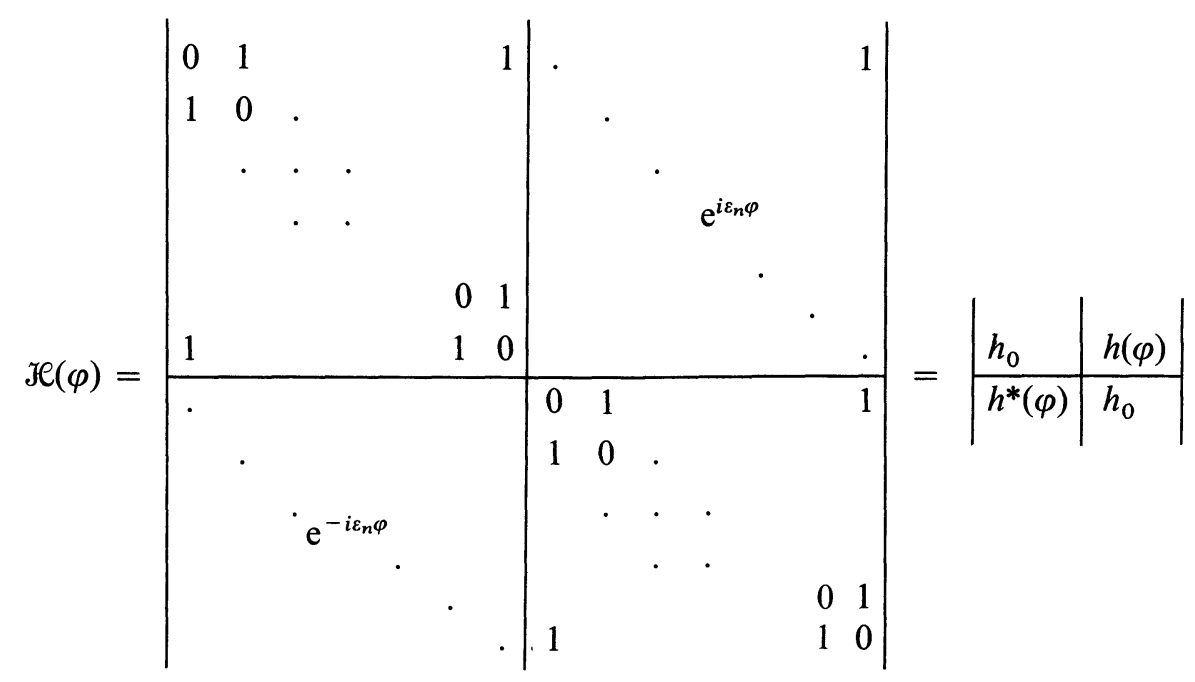

with, for instance, $0<\varphi<\pi$. 
$\mathscr{H}(\varphi)$ describes a 1-dimensional system consisting of 2 identical chains $\left(\mathrm{C}_{1}\right)$ and $\left(\mathrm{C}_{2}\right)$ of $N$ sites, with a constant coupling (taken as unity) between adjacent sites $\left(N \times N\right.$ submatrices $\left.h_{0}\right)$, and an interaction (submatrix $h(\varphi)$ with matrix elements $\mathrm{e}^{i \varepsilon_{n} \varphi}$ ) between sites (numbered $n$ ) facing each other on $\left(C_{1}\right)$ and $\left(C_{2}\right)$. A conventional representation is drawn in figure 3 , with right (resp. left) arrows for $\varepsilon_{n}=+1$ (resp. $\varepsilon_{n}=-1$ ) on the $n$th rung.

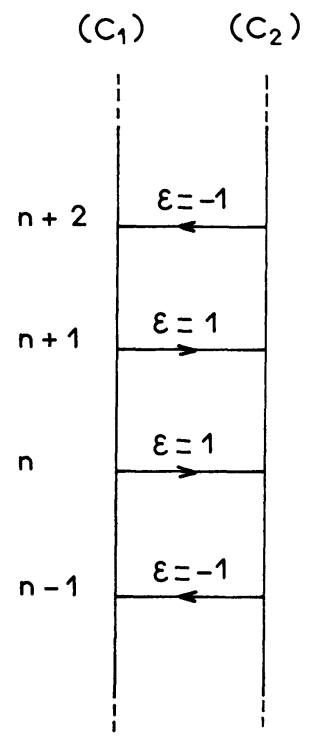

FIG. 3. - Oriented ladder obtained after the Bloch transformation.

Let $n(E, \varphi)$ be the DOS per atom for $\operatorname{He}(\varphi),(n(E, \varphi)$ is normalized to unity). The total DOS for the 2dimensional system is

$$
N(E)=\frac{1}{\pi} \int_{0}^{\pi} n(E, \varphi) \mathrm{d} \varphi .
$$

In the case of disordered networks, we are interested in the ensemble averaged DOS which we write as $\langle n(E, \varphi)\rangle$ and $\langle N(E)\rangle$ :

$$
\langle N(E)\rangle=\frac{1}{\pi} \int_{0}^{\pi}\langle n(E, \varphi)\rangle \mathrm{d} \varphi .
$$

\subsection{Calculation of THE moments. - Let}

$$
\mu_{n}(\varphi)=\int\langle n(E, \varphi)\rangle E^{n} \mathrm{~d} E
$$

be the moments of the average DOS of the hamiltonian $\operatorname{He}(\varphi)(1)$, and

$$
\begin{aligned}
\mu_{n} & =\int\langle N(E)\rangle E^{n} \mathrm{~d} E \\
& =\frac{1}{\pi} \int_{0}^{\pi} \mu_{n}(\varphi) \mathrm{d} \varphi
\end{aligned}
$$

the corresponding moments of the total average DOS. We may write

$$
\mu_{n}(\varphi)=\left\langle\left(\mathscr{H}(\varphi)^{n}\right)_{0,0}\right\rangle
$$

where the index 0 refers to some site taken as the origin (the translational invariance for the ensemble averaged DOS allows us to restrict the consideration to one particular site).

Eq. (6) shows that, as usual within the tight-binding approximation, the $n$th moment is associated with a sum over all closed cycles with $n$ steps, which can be drawn on the graph of figure 3, starting and ending at site 0 . Each visited bond introduces a factor equal to the corresponding hamiltonian matrix element, that is either 1 (bond belonging to $\left(C_{1}\right)$ or $\left(C_{2}\right)$ ) or $\mathrm{e}^{ \pm i \varepsilon_{m} \varphi}$ (if the bond is along the $m$ th rung, the \pm sign depending on whether one goes from $\left(C_{1}\right)$ towards $\left(C_{2}\right)$ or vice versa). Eq. (6) then takes the form

$$
\mu_{n}(\varphi)=\left\langle\sum_{\substack{n \text {-step } \\ \text { cycles }}} \exp i\left(\sum_{\substack{\text { visited } \\ \text { rungs }}} \pm \varepsilon_{m} \varphi\right)\right\rangle
$$

which, after integration over $\varphi$, yields :

$$
\mu_{n}=\left\langle\sum_{\substack{n \text {-step } \\ \text { cycles }}} \delta\left(\sum_{\substack{\text { visited } \\ \text { rungs }}} \pm \varepsilon_{m}\right)\right\rangle .
$$

At this level, we clearly see how the peculiarities of our model simplify the evaluation of the exact ensemble averaged moments. Let $p_{m}$ be the algebraic number of visits to the $m$ th rung in a given cycle. In order to calculate the moments $\mu_{n}$, we only have to enumerate the closed cycles and weight each of them by the probability for the corresponding sum $\left(\Sigma_{m} p_{m} \varepsilon_{m}\right)$ to be zero.

Actually, tree cycles, i.e. cycles where all bonds are systematically described in both directions, automatically lead to a zero value of $\left(\Sigma_{m} p_{m} \varepsilon_{m}\right)$. The evaluation of $\mu_{n}$ is then carried out by computing first the weighted number of non self-retracing cycles and by adorning them afterwards with tree cycles [17].

2.3 DERIVATION OF THE DENSITY OF STATES FROM THE MOMENTS. - An approximate $\operatorname{DOS}\langle N(E)\rangle$ may be obtained from the moments $\mu_{n}$ by a variety of techniques. Let $\langle G(E)\rangle$ be the average Green's function of the hamiltonian $\mathscr{H}$ with

$$
\langle N(E)\rangle=\frac{1}{\pi} \operatorname{Im}\left\langle G(E)_{0,0}\right\rangle .
$$

We express $\left\langle G(E)_{0,0}\right\rangle$ as a continued fraction [12-14]

$$
\left\langle G(E)_{0,0}\right\rangle=\frac{1}{E-\frac{b_{1}}{E-\frac{b_{2}}{E-.} \cdot}}
$$


where $b_{p}$ is a rational function of the $p$ first non-zero moments. These coefficients are known to converge towards a limit $b_{\infty}$ related to the band edge energy $E_{M}$ by :

$$
E_{M}=2 b_{\infty}^{1 / 2} .
$$

(In our case, $\left|E_{M}\right|=Z=3$, and $b_{\infty}=9 / 4=2.25$.) Actually, the coefficients $b_{n}$ are seen to oscillate around this limit (Fig. 4), and their convergence is far more rapid for the averaged disordered networks than for the HL regular lattice. This confirms an earlier observation of Gaspard for 3-dimensional systems $[13,14]$.

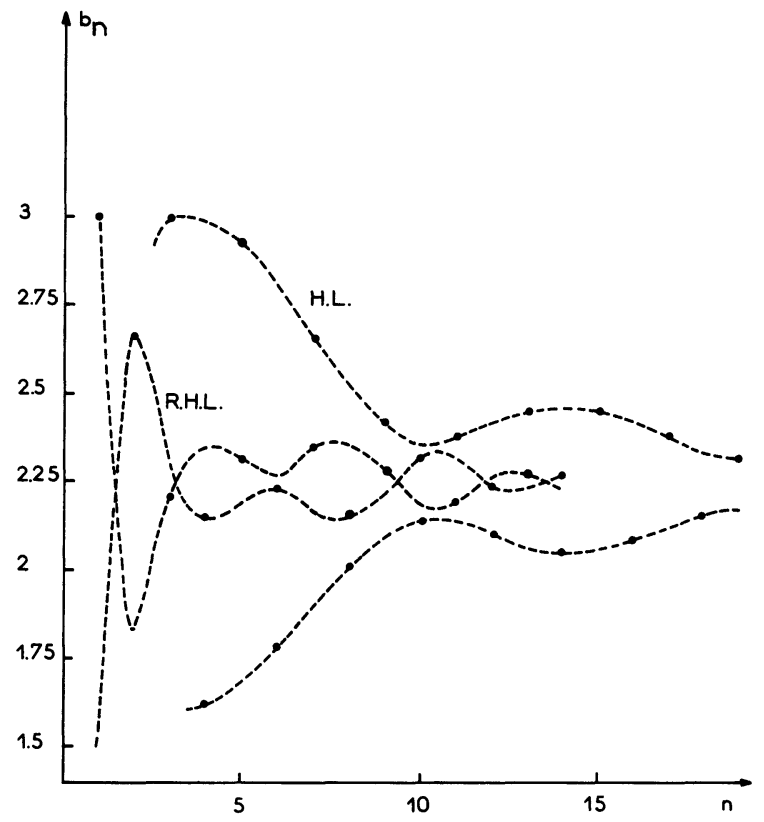

Fig. 4. - Continued fraction coefficients for the honeycomb and random honeycomb lattices.

Only the 14 first $b_{n}$ 's were exactly computed. As can be seen from figure 4 , it would be difficult to extrapolate safely the oscillations thus obtained for $n \geqslant 15$. For this reason we simply adopted $b_{n}=b_{\infty}$ in this region, keeping in mind that this procedure is likely to reduce the sharpness of the singularities of $\langle N(E)\rangle$. The average DOS thus obtained is shown in figure 5. Its most noteworthy features are :

i) the presence of a maximum near $|E|=0.75$ (peak A),

ii) a secondary maximum near $|E|=1.65$ (peak B), and

iii) a sharp maximum near $|E|=2.82$ very close to the band edge (peak C). Also shown on the left part of figure 5 are the DOS obtained with only the first 6 and 8 correct $b_{n}$; the gradual appearance of the characteristic structures is clear.

In order to check the foregoing results, we carried out a direct calculation of $\langle N(E)\rangle$ over an ensemble of randomly generated structures satisfying the requi-

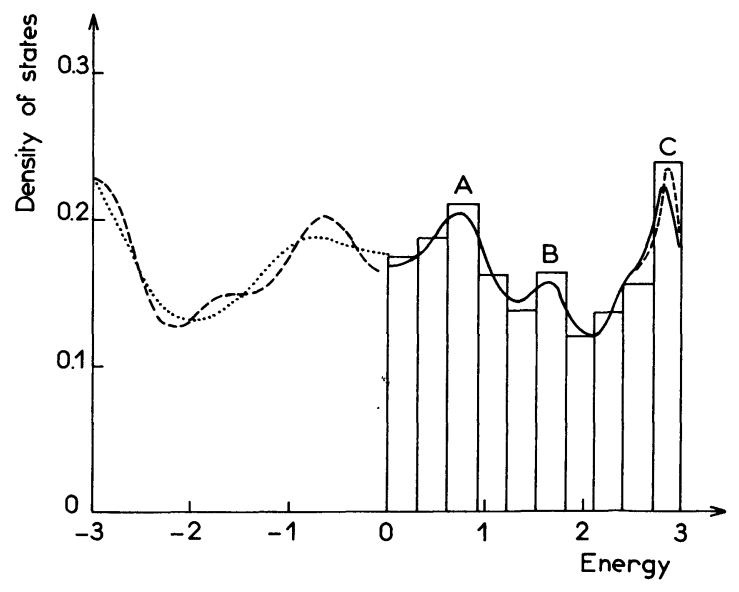

FIG. 5. - Average density of states for the random honeycomb lattice : - Left part : calculated with 6 moments (dotted line) and 8 moments (dashed line). - Right part : calculated with 14 moments ; directly computed histogram ; (dotted line : first appearance of peak (C) with 10 moments).

rements of section 2.1. We obtained the histogram drawn in figure 5 , which essentially confirms the moment method. In particular, we may notice the actual appearance of a secondary maximum (B) for $1.5<|E|<1.8$ and of a higher peak (Ä) for $0.6<|E|<0.9$. (The rather large histogram interval $\Delta E=0.3$ to which we restricted ourselves in this check does not permit one to decide whether there actually appears a maximum, (peak (C)), close to the band edge, or whether the maximum is obtained $a t$ the band edge. Nevertheless it is certain that the general trend is an increase of $\langle N(E)\rangle$ between, say, $|E|=2.1$ and $|E|=3$, and that the band edge is actually obtained for $|E|=E_{M}=Z=3$.)

We now account for the structures appearing in the $\langle N(E)\rangle$ plot.

i) If we exclude the vicinity of the band edges, the general shape of the DOS is determined by the local order, that is by the configuration of the rungs in the neighbourhood of each site of figure 3. This means that the first moments are sufficient to determine the essential features of $\langle N(E)\rangle$ inside the band; (actually, we may notice that peaks (A) and (B) in figure 5 appear as soon as 8 even order moments are taken into account). Moreover, among the local configurations of rungs, an essential role is played by clusters of contiguous rungs with the same orientation, since such clusters may accommodate a large number of closed paths winding around some simple loops inside the cluster and thus yield an important contribution to the moments $\left({ }^{1}\right)$.

In order to appreciate the influence on the ensemble averaged DOS of such clusters, we may consider

( $\left.{ }^{1}\right)$ We may also remember that we are dealing here with a kind of 1-dimensional disorder; it is well-known $[15,16]$ that the main accidents in the DOS are then associated with particular configurations of contiguous elementary units, that is, in our case, with clusters of adjacent rungs with the same orientation. 


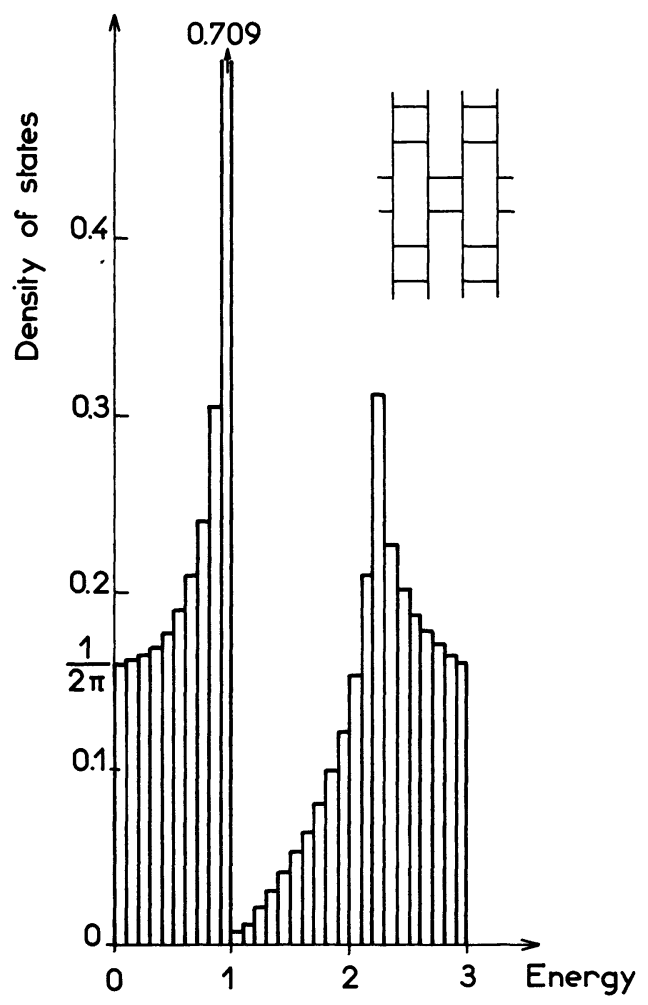

FIG. $6 a$. - Density of states for the 4.8 lattice.



FIG. $6 b$. - Density of states for the 4.4.10 lattice.

lattices which contain only clusters of a given number of rungs. These lattices are periodic both along the $x$ - and $y$-directions. The singularities which show up in their DOS are likely to reappear in the ensemble averaged DOS, but they are weighted by the probability for the cluster to be present in the random network.
This probability is itself a rapidly decreasing function of the length of the cluster (i.e. of the number of rungs it contains), so that only the smallest clusters have to be considered. They correspond to the following lattices :

- 4-8 lattice [18] (Fig. 6a),

- 4-4-10 lattice (Fig. 6b).

Comparing figure 5 with figure 6 , we realize :

- that peak (A) recalls the (inverse square-root) divergence for $|E|=1$ which appears in the DOS of the 4-8 lattice (its shift towards lower $|E|$ values can be attributed to the asymmetry of both curves $6 a$ and $6 b$ in the neighbourhood of $|E|=1$ ),

- that peak (B) is to be associated with the (logarithmic) singularity for $|E|=1.56$ of the 4-4-10 lattice DOS.

ii) In order to obtain a reasonably precise description of the DOS close to the band edges, it is necessary to use the information which is given by high order moments : for instance, it appears in figure 5 that the ultimate peak (C) only shows up when at least $10 b_{n}$ (or 10 non-zero moments) are calculated exactly.

In this context, ladders, that is clusters of many contiguous rungs with the same orientation are particularly important since they give large contributions to moments of high order. For the infinite ladder (Fig. 7), the DOS has an inverse square-root divergence at the band edge, as could be expected for such a one-dimensional system. It is reasonable to assume that long clusters of finite length will still lead to a maximum close to the band limit; (this view is supported by the observation of figure $6 a$ and $6 b$, where such maxima already appear, and come nearer

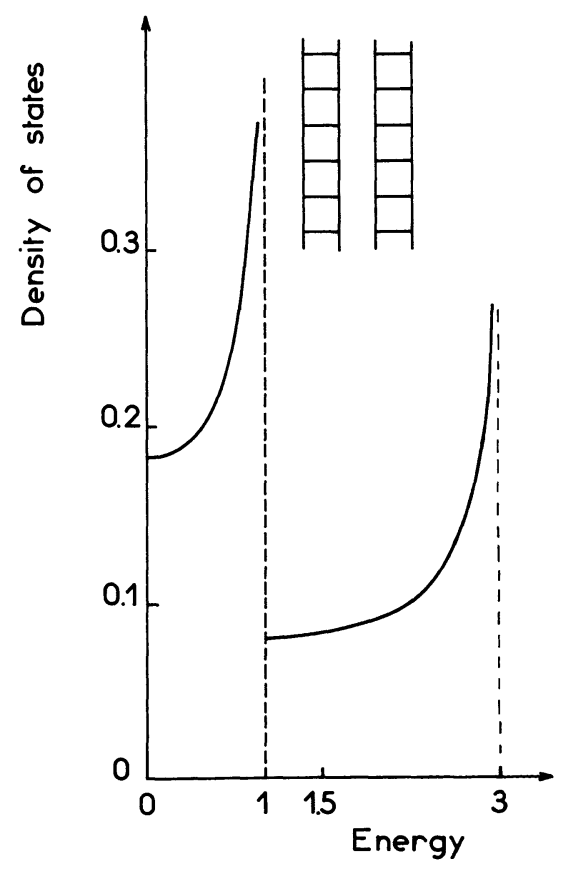

FIG. 7. - Density of states for the infinite ladder. 
to $|E|=3$ when the length of the cluster is increased from 2 to 3.) Averaging over the contributions of clusters of various lengths, weighted by their respective probability of occurrence, should thus lead to peak (C). The presence of this DOS maximum near the band limit is further supported by the CPA calculation described in the Appendix.

This rather complete study of a particular class of 2-dimensional partially topologically disordered systems has given us the opportunity to introduce the main ideas and methods which will prove useful in the study of the corresponding 3-dimensional networks, to which the next section is devoted.

3. Three-dimensional systems. - 3.1 General fEATURES OF THE STUDIED NETWORKS. - We now deal with 4-fold coordinated $(Z=4)$, 3-dimensional networks and submit them to the same general conditions as the previously studied 2-dimensional systems : essentially, we keep partial periodicity and introduce disorder only along either 1 or 2 directions in space.

Consider for instance the wurtzite structure (Fig. 8). (It is recalled that this lattice is a polytype of the blende or diamond lattice, which has the same DOS within the restricted LCAO formalism we use here [19].) It is made up of horizontal hexagonal lattices composed of chair hexagons, connected by vertical bonds which point alternately in the upward or downward direction. Each hexagonal lattice may be flattened into a plane honeycomb lattice, and redrawn as in section 2 (Fig. 1a).

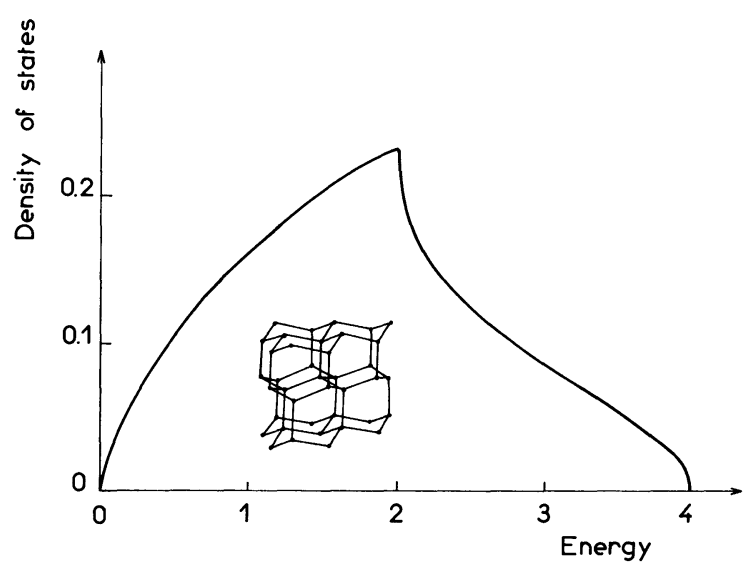

FIG. 8. - Wurtzite lattice and corresponding density of states.

Keeping a succession of such identical superposed HL planes, we may now introduce disorder by admitting the vertical bonds to be randomly distributed : once a choice has been made for the bonds starting from a particular HL plane, the $Z=4$ requirement suffices to determine all the bonds throughout the lattice. We obtain a first ensemble of networks with a 1-dimensional periodicity along the vertical $z$-direction and a 2-dimensional disorder (section 3.2).

We may go one step further and partially randomize the horizontal $\mathrm{HL}$ as in section 2, keeping, for instance, a systematic bonding of atoms along chains parallel to the $y$-direction and distributing randomly the connections parallel to the $x$-axis. However, in order to simplify the calculations, we shall restrict ourselves to the case where an overall periodicity is kept both along the $x$ - and $z$-directions. We then obtain a second ensemble of networks with a 1-dimensional disorder (along the $y$-direction) and a 2-dimensional periodicity (section 3.3).

It is obvious that both procedures may be considered as resulting from the suppression of 2 bonds per atom out of 6 in a simple cubic lattice (systematically maintaining 2 bonds per atom parallel to the $y$-axis).

3.2 FirST CASE : 2-DIMENSIONAL DISORDER. As in section 2, we make use of the periodicity (along the $z$-axis) with 2 consecutive HL as a unit cell (cf. Fig. 9) to make a Bloch transformation. We thus obtain a series of subhamiltonians

$$
\tilde{\mathscr{H}}(\varphi)=\left|\begin{array}{|l|l|}
\tilde{h}_{0} & \tilde{h}(\varphi) \\
\hline \tilde{h}^{*}(\varphi) & \tilde{h}_{0}
\end{array}\right|
$$

where $\varphi$ is a $k$-vector parallel to the $z$-axis; $\tilde{h}_{0}$ is the hamiltonian for a HL plane; $\tilde{h}(\varphi)$ is a diagonal matrix with elements $\mathrm{e}^{i \varepsilon_{n} \varphi}=\mathrm{e}^{ \pm i \varphi}$ (+ corresponding to an upward bond, - corresponding to a downward bond), in complete analogy with what we have found in section 2.

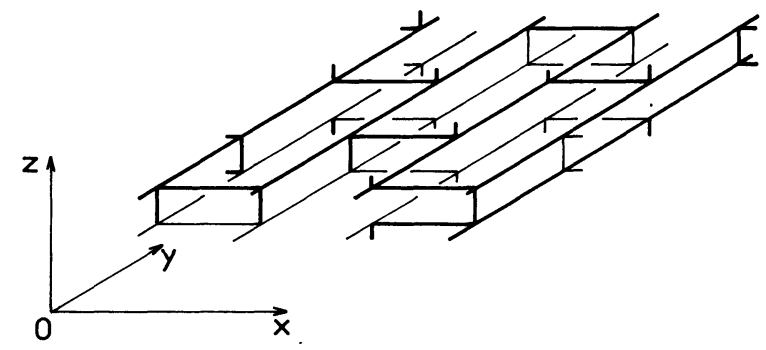

FIG. 9. - Unit cell for a 3-dimensional random network with 1-dimensional periodicity along the $z$-direction.

The ensemble averaged moments may then be calculated as above : closed paths are enumerated for a 2-dimensional network which here consists of two horizontal $\mathrm{HL}$ with oriented vertical bonds between sites facing one another, and each path is weighted by the probability for $\left(\Sigma_{m} p_{m} \varepsilon_{m}\right)$ (where $p_{m}$ is the algebraic number of visits to the $m$ th bond) to be zero.

From these moments, one can as above deduce the continued fraction coefficients $b_{n}$ : these have been calculated up to $n=11$; they oscillate around the limit value $b_{\infty}=4$. While the amplitude of the oscillations is much reduced when compared with the wurtzite (diamond) case, the last oscillation obtained is still quite important, and, again, it is difficult to extrapolate safely for higher $n$. Adopting $b_{n}=b_{\infty}(n \geqslant 12)$, we obtain the DOS shown in 
figure 10. Two peaks appear : for $E=0$ and $|E|=2$; (possible shoulders may be present at $|E|=3$ and $|E|=3.7)$.

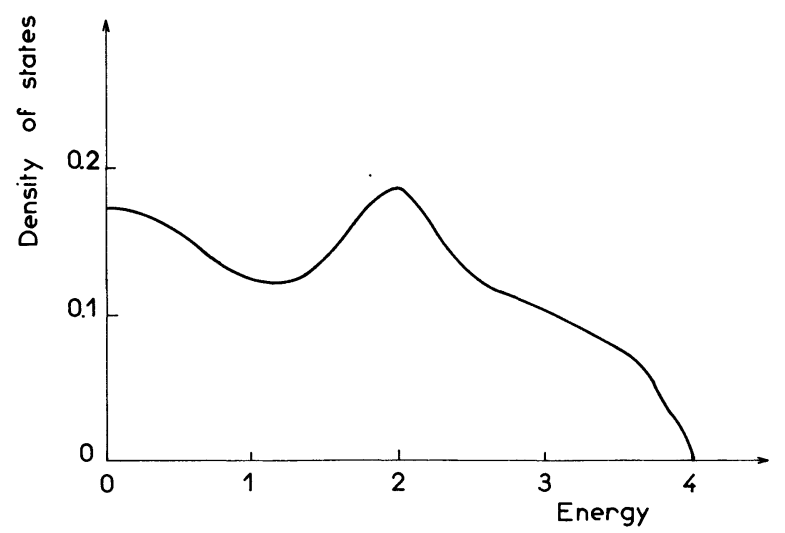

FIG. 10. - Average density of states for the random lattice of figure 9.

Our interpretation will be based on the same principles as in section 2 . Of primary importance are

i) periodic lattices with clusters of identically oriented vertical bonds: we only considered the simplest one (Fig. 11), the DOS of which shows two marked peaks for $E=0$ and $|E|=2$,

ii) networks with reduced dimensionality : here, they are obtained when, in a large region of a horizontal plane, all bonds have the same orientation, yielding a 2-dimensional double honeycomb lattice (DOS in figure $15 a)\left({ }^{2}\right)$.

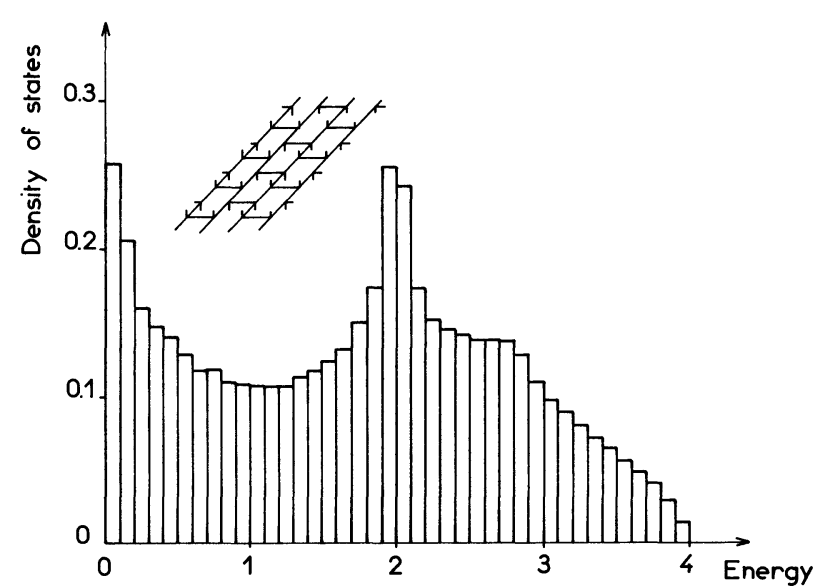

FIG. 11. - A completely periodic lattice belonging to the class defined by figure 9 and the corresponding density of states.

$\left(^{2}\right)$ Whenever one considers such a sandwich network, consisting of a superposition of two identical 2-dimensional lattices with perpendicular bonds between equivalent atoms on these lattices, it is readily shown that the DOS has the general form

$$
N_{3}(E)=\frac{1}{2}\left(N_{2}(E+1)+N_{2}(E-1)\right)
$$

where $N_{2}$ and $N_{3}$ refer to the isolated 2-dimensional lattice and the sandwich respectively. In particular, for the double honeycomb lattice (DHL), we have

$$
N_{\mathrm{DHL}}(E)=\frac{1}{2}\left(N_{\mathrm{HL}}(E+1)+N_{\mathrm{HL}}(E-1)\right) .
$$

All these networks show singularities for $|E|=2$ and $E=0$. It is thus reasonable that the ensemble averaged DOS for the random networks show essentially two peaks centered on these particular energies. Despite the limited number of moments we could use, we can have fair confidence in the reality of both these structures.

3.3 SECOND CASE : 1-DIMENSIONAL DISORDER. 3.3.1 We now deal with a second class of partially disordered networks which can be viewed as follows :

- all atoms are located at the vertices of a simple cubic lattice, the three coordinates of which are taken as integers,

- every atom is linked to its two neighbours along the $y$-axis, so that again we have chains of atoms parallel to the $y$-direction,

- in a given plane $y=n$ (perpendicular to the chains), every atom is linked to two out of its four neighbours (so that a constant coordination number $Z=4$ is maintained), and the whole bond configuration is periodic both along the $x$ - and $z$-directions, with a square unit cell of side equal to 2 (figure 12 shows 5 possible arrangements of the bonds within the unit cell; the 11 other ones are obtained by translating the graphs of figure 12 with respect to the unit cell),

- the configurations of the different planes $y=n$ are chosen independently among all possible ones, and randomly distributed.

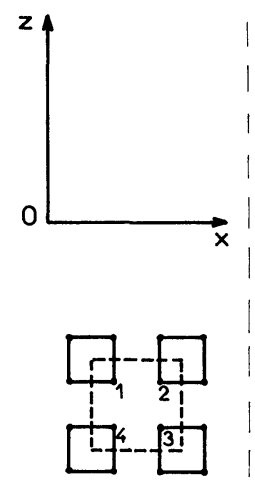

(a)

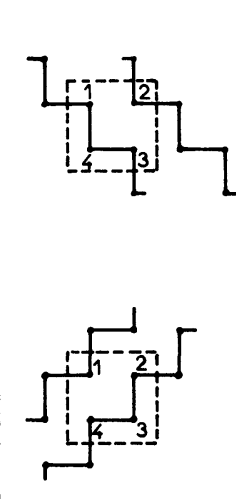

(b)



(c)
FIG. 12. - Five possible configurations of the $y=n$ plane for random systems with 2 -fold periodicity along the $x$ - and $z$-axes.

As was indicated previously, we obtain a class of networks with 1-dimensional disorder (along the $y$-axis) and twofold periodicity (along both $x$ - and $z$-directions). We take advantage of this periodicity to perform a double Bloch transformation with two wave-vector-like parameters $\varphi$ and $\psi$, which gives a series of subhamiltonians $\operatorname{He}(\varphi, \psi)$. These may be interpreted as describing a pipe (Fig. 13) made up of four identical linear chains along the 

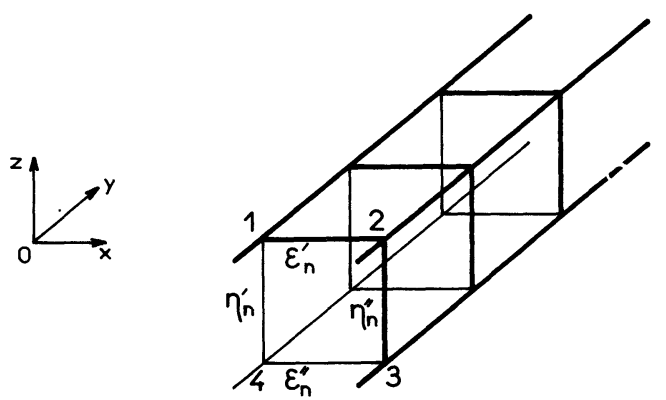

FIG. 13. - Pipe with oriented rungs obtained after a Bloch transformation.

$y$-direction. For a given value of $y,(y=n)$, the four sites (one on each chain) interact via matrix elements :

$\left.\langle 1|\operatorname{He}(\varphi, \psi)| 2\rangle=\exp \left(i_{n}^{\prime} \varphi\right)=\langle 2|\operatorname{He}(\varphi, \psi)| 1\rangle^{*}\right)$ $\langle 4|\operatorname{He}(\varphi, \psi)| 3\rangle=\exp \left(i_{n}^{\prime \prime} \varphi\right)=\langle 3|\operatorname{He}(\varphi, \psi)| 4\rangle^{*}$ $\langle 1|\operatorname{Te}(\varphi, \psi)| 4\rangle=\exp \left(i \eta_{n}^{\prime} \psi\right)=\langle 4|\operatorname{He}(\varphi, \psi)| 1\rangle^{*}$ $\langle 2|\operatorname{He}(\varphi, \psi)| 3\rangle=\exp \left(i \eta_{n}^{\prime \prime} \psi\right)=\langle 3|\operatorname{He}(\varphi, \psi)| 2\rangle^{*}$

with $\varepsilon_{n}^{\prime}= \pm 1$ depending on whether sites 1 and 2 in the unit cell are linked or not, and analogous conventions for $\varepsilon_{n}^{\prime \prime}, \eta_{n}^{\prime}$ and $\eta_{n}^{\prime \prime}$.

To the random distribution, with equal probabilities, of the 16 configurations of planes perpendicular to the $y$-axis, correspond random, uncorrelated distributions of the four parameters $\varepsilon_{n}^{\prime}, \varepsilon_{n}^{\prime \prime}, \eta_{n}^{\prime}, \eta_{n}^{\prime \prime}$ among their two possible values.

3.3.2 The general techniques used above to compute the average moments may be resumed here; every closed circuit described on the pipe of figure 13 must be weighted according to the probability for $\left(\Sigma_{m}\left(p_{m}^{\prime} \varepsilon_{m}^{\prime}+p_{m}^{\prime \prime} \varepsilon_{m}^{\prime \prime}\right)\right)$ and $\left(\Sigma_{m}\left(q_{m}^{\prime} \eta_{m}^{\prime}+q_{m}^{\prime \prime} \eta_{m}^{\prime \prime}\right)\right)$ to be zero, where the $p$ 's and $q$ 's are the algebraic numbers of visits to the various bonds. The 9 first coefficients for the continued fraction describing $\langle N(E)\rangle$ have been computed. Prolonging them by their limit value $b_{\infty}=4$, we obtain an approximate DOS shown in figure 14, with peaks for $E=0,|E|=1.75$ and $|E|=3.5$.

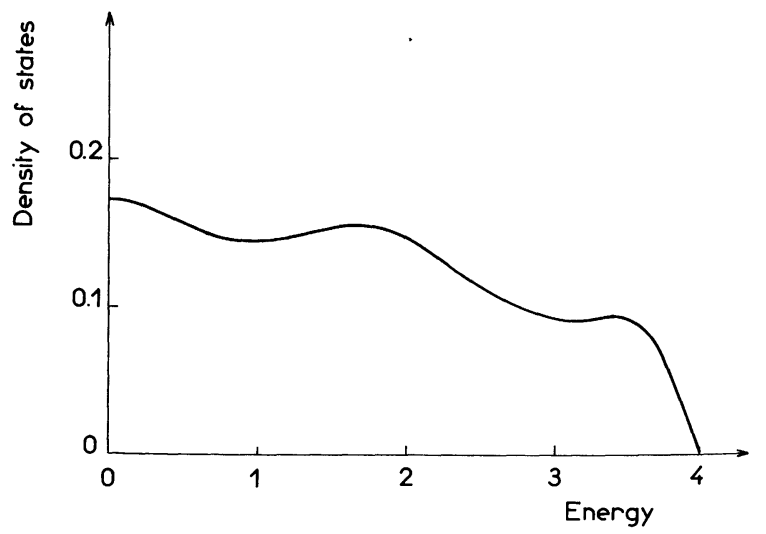

FIG. 14. - Average density of states for the random networks with 2 -fold periodicity.
As before, we try to account for these peaks by referring to particular lattices or clusters. Of particular importance are those of reduced dimensionality which may be here

i) 1-dimensional : by piling up identical square planes (Fig. 12a) one gets square pipes; while their occurrence is of low probability, such clusters can play a non-negligible role because of the strong singularities which appear in their DOS. For infinite pipes, the DOS contains divergencies at $E=0,|E|=2$ (with a dissymmetrical shape) and $|E|=4$ (Fig. 15b),

ii) 2-dimensional, with a variety of possible configurations :

- piling up square planes (Fig. 12a), with a systematic shift between two consecutive planes, leads to the double honeycomb lattice, already mentioned in section 3.2 (DOS of figure 15a),

- piling up randomly two out of the four types of square planes (deduced from figure $12 a$ by translating the squares with respect to the unit cell), leads to the double random honeycomb lattice (DRHL); from eq. (13) it is readily seen that the DOS of this lattice (Fig. 15c) is given by

$$
N_{\mathrm{DRHL}}(E)=\frac{1}{2}\left(N_{\mathrm{RHL}}(E+1)+N_{\mathrm{RHL}}(E-1)\right)
$$

where $N_{\mathrm{RHL}}(E)$ is the DOS of the two-dimensional random honeycomb lattice (Fig. 5),

- piling up identical zigzag planes (Fig. 12b) or identical square saw-tooth planes (Fig. 12c) leads to simple quadratic lattices with DOS's having a logarithmic divergence at $E=0$.

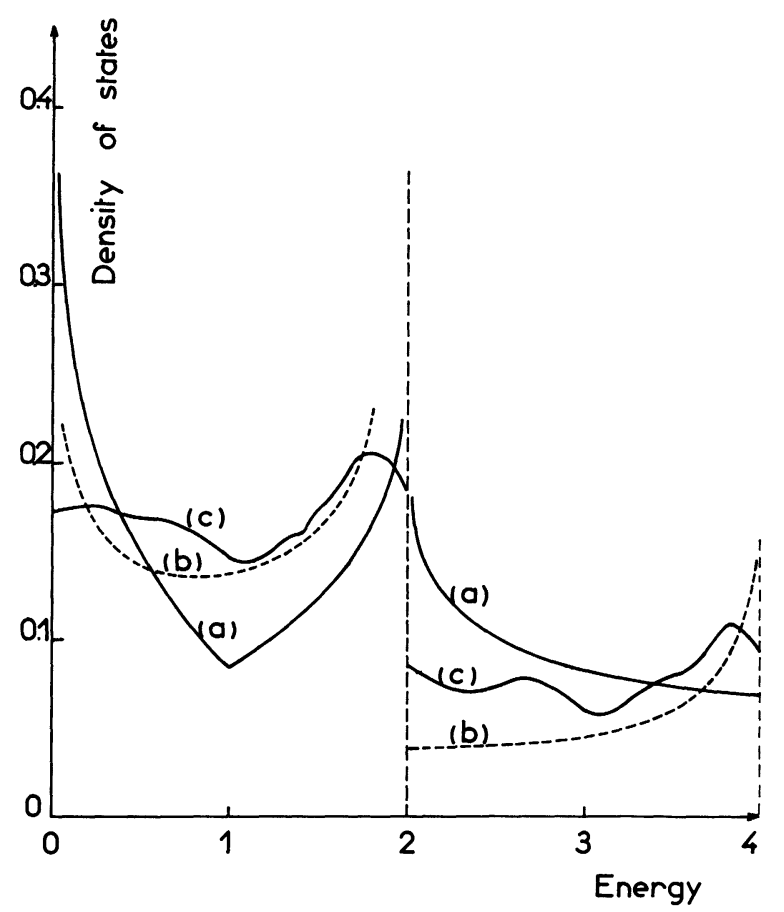

FIG. 15. - a) Density of states for the double honeycomb lattice. b) Density of states for the infinite square pipe. c) Density of states for the double random honeycomb lattice. 
All the lattices described above have a DOS showing a maximum for $E=0$ and for $|E| \lesssim 2$ (with an asymmetry which favors the $|E|<2$ region in the case of figures $15 b-15 c$ ). They account for the peaks at $E=0$ and $|E|=1.75$ in the average DOS (Fig. 14) of the random networks considered in this section.

As for the last maximum near the band edge which appears in figure 14, it should be related to the possible occurrence of pipes (DOS in figure 15b) in the studied random networks; these pipes with their DOS inverse square-root divergence for $|E|=4$ play the same role here as did the ladders for the 2-dimensional RHL. This interpretation is further supported by the following results :

i) if we randomly pile up only zigzag $x \mathrm{O} z$ planes (Fig. 12b), thus excluding the presence of pipes inside the random networks, the DOS obtained (Fig. 16a) lacks the band edge peak;

ii) if we randomly pile up only square $x \mathrm{O} z$ planes (Fig. 12a), which enhances the probability for pipes to appear, we recover this peak (Fig. 16b) and it is, as expected, more pronounced than on figure 14 .

Again, we can easily obtain a simple explanation of the most noteworthy features of the average DOS by reference to some remarkable lattices.

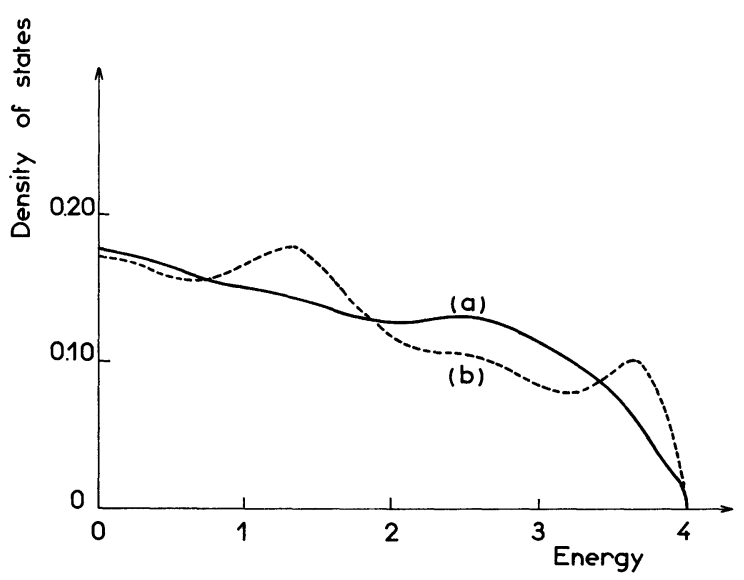

FIG. 16. - a) Average density of states for a random network made up of zigzag (Fig. 12b). b) Average density of states for a random network made up of squares (Fig. 12a).

4. Conclusion. - Through the evaluation of exact average moments and the use of a continued fraction method - which confirms itself as providing a well-adapted tool for this kind of problem -, approximate densities of states have been computed for a class of partially periodic, partially disordered networks.

Their DOS show a number of structures : they can be systematically related to the DOS singularities of a few types of lattices which may appear, at least locally, in the ensemble of networks under study; among these lattices, those of reduced dimensionality (e.g. 2-dimensional lattices inside a 3-dimensional network) are of special importance, since they lead to stronger singularities.

The peculiar geometrical features of our model networks make it possible to vary easily the probability of occurrence of these local configurations, and thus offer an opportunity to check their influence on the average DOS. This flexibility should prove useful for future work : the next step would be, while maintaining partial periodicity, to complicate the unit cell somewhat, in order to remove some particularly unrealistic properties of the networks studied above (for instance, the presence of 4-fold rings, which are responsible for the peaks of $\langle N(E)\rangle$ that are frequently observed for $E=0$ ).

\section{APPENDIX}

Application of the CPA to the networks under study (2-dimensional case). - One may notice that the partial periodicity which is kept in our models makes it possible to transform their topological disorder into a kind of compositional disorder, which appears, after the Bloch transformation, in the random values of the matrix elements of the subhamiltonians $h(\varphi)$. The average DOS of our networks can thus be approached using the analytical approximations which have been devised to deal with compositional disorder. Here we briefly show how the CPA $[20,21]$ can be applied to the 2-dimensional systems of section 2 .

With a simple unitary transformation, the hamiltonian (1) can be put in the following form

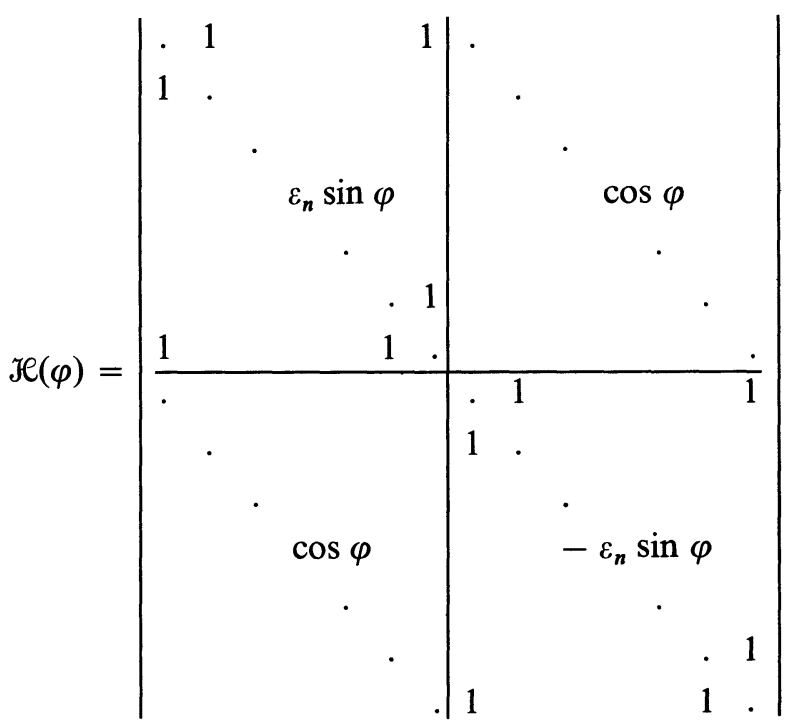

(A.1)

Eq. (A.1) can be interpreted as describing a ladder (Fig. 17a) where the disorder affects the diagonal matrix elements $\left( \pm \varepsilon_{n} \sin \varphi\right)$ corresponding to the two sites facing each other on each rung.

Following the CPA scheme, we define an effective hamiltonian $\mathscr{H}^{*}$ with diagonal matrix elements $\sigma=\sigma(E, \varphi)$ on all sites and off-diagonal matrix elements $\sigma^{\prime}=\sigma^{\prime}(E, \varphi)$ between the two sites of each rung: 


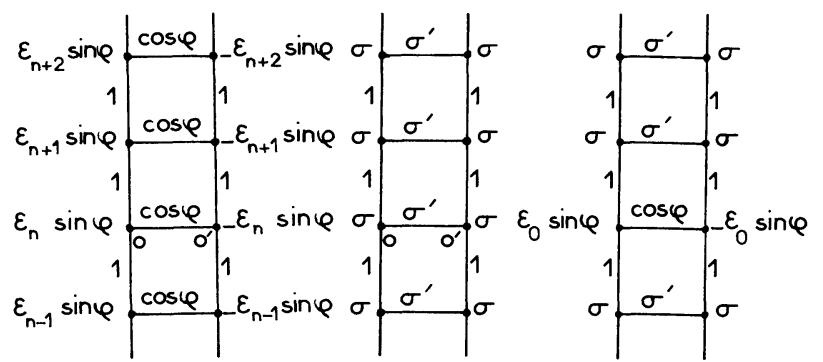

FIG. 17. - a) Ladder of figure 3 after a unitary transformation. b) Effective ladder within the coherent potential approximation. c) One rung embedded in the effective ladder.

$H^{*}$ describes the effective ladder shown on figure $17 b$. We then isolate one rung and the corresponding pair of sites, say 0 and $0^{\prime}$, on the true ladder, and embed it in the effective ladder, thus obtaining the system shown in figure $17 c$. Let $g^{*}$ and $g$ be the $2 \times 2$ matrix propagators, restricted to sites 0 and $0^{\prime}$, corresponding respectively to the graphs of figures $17 b$ and $17 c$. $\sigma$ and $\sigma^{\prime}$ are determined by the CPA condition :

$$
\langle g\rangle=g^{*}
$$

where $\langle g\rangle$ is the average value of $g$ (over the two possible values of $\varepsilon_{0}$ ).

For the periodic effective ladder, one readily finds :

with

$$
g^{*}(E, \varphi)=\left|\begin{array}{cc}
a(E, \varphi) & a^{\prime}(E, \varphi) \\
a^{\prime}(E, \varphi) & a(E, \varphi)
\end{array}\right|
$$

$$
\begin{aligned}
& a(E, \varphi)=\frac{1}{2}\left\{\left[\left(E-\sigma-\sigma^{\prime}\right)^{2}-4\right]^{-1 / 2}+\right.\left.+\left[\left(E-\sigma+\sigma^{\prime}\right)^{2}-4\right]^{-1 / 2}\right\} \\
& a^{\prime}(E, \varphi)=\frac{1}{2}\left\{\left[\left(E-\sigma-\sigma^{\prime}\right)^{2}-4\right]^{-1 / 2}-\right. \\
&\left.-\left[\left(E-\sigma+\sigma^{\prime}\right)^{2}-4\right]^{-1 / 2}\right\}
\end{aligned}
$$

Comparing figures $17 b$ and $17 c$, we see that $g$ and $g^{*}$ are related through the following equation :

$$
g=g^{*}\left[1-\left(V_{0}-\Sigma\right) g^{*}\right]^{-1}
$$

with

$$
\begin{aligned}
\Sigma & =\left|\begin{array}{ll}
\sigma & \sigma^{\prime}-\cos \varphi \\
\sigma^{\prime}-\cos \varphi & \sigma
\end{array}\right| \\
V_{0} & =\varepsilon_{0}\left|\begin{array}{cc}
\sin \varphi & 0 \\
0 & -\sin \varphi
\end{array}\right| .
\end{aligned}
$$

The CPA condition (A.2) thus leads to

$$
\left\langle\left[1-\left(V_{0}-\Sigma\right) g^{*}\right]^{-1}\right\rangle=1 .
$$

Developing (A.7) in powers of $V_{0}$, and noticing that

$$
\left\langle\left(\varepsilon_{0}\right)^{n}\right\rangle=\left\{\begin{array}{l}
0 \text { if } n \text { is odd } \\
1 \text { if } n \text { is even }
\end{array}\right.
$$

we find

$$
\Sigma=V_{0} g^{*}\left(1+\Sigma g^{*}\right)^{-1} V_{0} .
$$

With the help of (A.8), we may express the coherent potentials $\sigma$ and $\sigma^{\prime}$ in terms of $g^{*}$ (A.3) :

$$
\left.\begin{array}{rl}
\sigma & =a\left[\sin ^{2} \varphi-\left(\sigma^{2}-\left(\sigma^{\prime}-\cos \varphi\right)^{2}\right)\right] \\
\operatorname{s} \varphi & =-a^{\prime}\left[\sin ^{2} \varphi-\left(\sigma^{2}-\left(\sigma^{\prime}-\cos \varphi\right)^{2}\right)\right]
\end{array}\right\}
$$

Solving eq. (A.4) and (A.9) together yields the CPA Green's function $g^{*}(E, \varphi)$, and the DOS :

$$
n_{\mathrm{CPA}}(E, \varphi) \equiv \frac{1}{\pi} \operatorname{Im} a(E, \varphi)
$$

This computation has been performed numerically. After integration over $\varphi$, one gets the total DOS $N_{\text {CPA }}(E)$ (Fig. 18).



FIG. 18. - Density of states for the random honeycomb lattice in the coherent potential approximation.

Comparing the result with figure 5 , we notice that the overall shape of the DOS inside the band is not correctly reproduced; peaks (A) and (B) do not show up; instead a logarithmic singularity is obtained for $|E|=1$. This lack of success is not really surprising : the CPA may be expected to give reasonable results for a two-component system, only in cases of small disorder, that is either when the concentration of one constituent is small, or when the matrix elements which introduce the disorder are small as compared to those which describe the interaction between atoms. Since both values \pm 1 of our disordered parameters $\varepsilon_{n}$ have the same probability, we are not in a situation where the first favorable factor might come into play. As for the second one, its role is 
restricted to the case $|\sin \varphi| \ll 1$. In other words, the CPA calculation is likely to give an accurate value $n_{\mathrm{CPA}}(E, \varphi)$ of the DOS, only for small values of $\varphi$. Except in the neighbourhood of the band edge, small values of $\varphi$ do not give a particularly important contribution to the integrated density of states $N_{\text {CPA }}$, and this quantity cannot be considered as a reliable approximation of the true DOS.

On the other hand, for $|E|$ close to $3, n_{\mathrm{CPA}}(E, \varphi)$ is different from zero only for small values of $\varphi$, and in this case, it may be expected to be reasonably exact. (In particular, for $\varphi=0$, the disorder vanishes, and we are left with a ladder DOS, which is, of course, exactly reproduced by the CPA.) We may thus assume that, in this energy range, the integrated density of states $N_{\mathrm{CPA}}$ is a fair approximation to the real DOS, and that the marked peak which appears for $|E|=2.8$ in figure 18 is not an artefact of the CPA, but reproduces an effective feature of the ensemble averaged DOS. This confirms the existence of peak (C) on figure 5, which showed up when a sufficient number of moments was taken into account.

\section{References}

[1] Weatre, D., Phys. Rev. Lett. 26 (1971) 1541

[2] Weaire, D. and Thorpe, M. F., Phys. Rev. B 4 (1971) 2508.

[3] Alben, R., Weaire, D. and Steinhardt, P., J. Phys. C 6 (1973) L 384, and references cited therein.

[4] John, W., Phys. Stat. Sol. 55 (1973) K 9.

[5] Streitwolf, H. W., Phys. Stat. Sol. 63 (1974) 529.

[6] Straley, J. P., Phys. Rev. B 6 (1972) 4086.

[7] Hulin, M., Phys. Stat. Sol. 52 (1972) 119.

[8] Lukes, T. and Nix, B., J. Phys. A 6 (1973) 1888.

[9] Friedel, J. and Lannoo, M., J. Physique 34 (1973) 483.

[10] Guttman, L., Phys. Rev. B 11 (1975) 764.

[11] Joannopoulos, J. D. and Yndurain, F., Phys. Rev. B 10 (1974) 5164, and references cited therein.

[12] Gaspard, J. P. and Cyrot-Lackmann, F., J. Phys. C 6 (1973) 3077.
[13] Gaspard, J. P., Proceedings of the Conference on Tetrahedrally Bonded Amorphous Semiconductors (Yorktown Heights 1974) AIP (1974) 170.

[14] Gaspard, J. P., Thèse de Doctorat d'Etat, Université d'Orsay (1975).

[15] HoRI, J., Spectral Properties of Disordered Chains and Lattices (Pergamon Press) 1968.

[16] Agacy, R. L. and Borland, R. E., Proc. Phys. Soc. 84 (1964) 1017.

[17] Roulet, B., Fisher, M. E. and Doniach, S., Phys. Rev. B 7 (1973) 403.

[18] Domв, C., Adv. Phys. 9 (1960) 149.

[19] ThORPE, M. F., J. Math. Phys. 13 (1972) 294.

[20] Soven, P., Phys. Rev. 156 (1967) 809.

[21] Velicky, B., KirkPatrick, S. and Ehrenreich, H., Phys. Rev. 175 (1968) 747. 